\section{Coming now to a chest clinic near you}

\author{
Charlotte E Bolton, ${ }^{1}$ Andrew Bush ${ }^{2}$
}

Premature birth means that instead of being fluid-filled and non-functional for many weeks, the immature lungs are gasfilled and have to support respiration. Often they are exposed to iatrogenic positive not physiological negative pressure expansion, infections, fluid overload and potentially hazardous treatments such as systemic steroids. Hence the respiratory sequelae of a premature birth need to be a major focus as more and more immature infants survive. Novel treatments, in particular, 'gentle' ventilator strategies (low mean airway pressures, faster rates), antenatal corticosteroids and inhaled surfactant have transformed the nature of the respiratory impairment from the traditional bronchopulmonary dysplasia (BPD) which was primarily an airways disease to 'new' BPD, more related to arrested alveolar development. However, our focus has long been on short term morbidity and mortality. As a community we have been particularly bad at following these young people up and appreciating their ongoing problems and risks. Specifically, paediatricians have sighed with relief when these children are no longer oxygen dependent, and have failed to keep these children under review. Furthermore, as we recently documented adult chest physicians rarely make enquiries about early life events. ${ }^{1}$ So survivors of prematurity are apt to disappear into a modern-day black hole.

In part, the problem may have arisen because of lack of appreciation of normal lung growth and the consequences of disease. There are three key stages which need to be optimal for long term lung health. First, lung growth in utero, ensuring the child is delivered with normally developed lungs, and second, the rate of lung growth in childhood, both determine whether the child will reach the full potential plateau at age $20-25$ years. ${ }^{2}$ The airway generations are laid down by 16 weeks and primitive gas exchanging units develop from around 24 weeks of

\footnotetext{
${ }^{1}$ Nottingham Respiratory Research Unit, University of Nottingham, Nottingham, UK; ${ }^{2}$ Royal Brompton \& Harefield NHS Foundation Trust, London, UK

Correspondence to Dr Charlotte E Bolton, Nottingham Respiratory Research Unit, University of Nottingham, Clinical Sciences Building, City Hospita Campus, Hucknall road, Nottingham NG5 1PB, UK; charlotte.bolton@nottingham.ac.uk
}

gestation. Numerous antenatal factors can affect lung development, and maternal smoking, which may have contributed significantly to the child being born prematurely will also damage the developing lungs before birth. Alveolarisation is a later phenomenon, commencing from 36 weeks and is especially active in the first months of life, exactly when the preterm infant is most likely to be exposed to insults such as hyperoxia, positive pressure ventilation and steroid therapy, all of which are toxic to the delicate developing gas-exchanging membrane. Finally, the rate of decline of lung function from this plateau will determine when the threshold for respiratory symptoms and disability will be reached. Decrements in early lung function can become magnified as the lung ages, producing a new population with premature airflow obstruction.

In this issue of Thorax, Kotecha et al ${ }^{3}$ report on a systematic review of the respiratory sequelae of prematurity from the age of five into adulthood. The manuscript covers a spectrum of gestational ages, and confirms that respiratory limitation is not solely an issue for the most extremely premature with BPD but is also important in children born $<37$ weeks without BPD (a very much larger group, obviously, than the extremely premature), and also in those extremely preterm children requiring no significant intervention. Thus, unless preterm and late-preterm delivery can be totally prevented, respiratory sequelae will be a problem for the foreseeable future. While there has been an apparent improvement in more recent BPD survivors compared with their predecessors, the potential for morbidity remains a huge concern. In this same issue, Vollsaetter and colleagues reported follow-up of two cohorts of subjects born premature ( $\leq 28$ weeks) or $\leq 1000$ g compared with matched control populations in order to determine both lung function but also any change in trajectory of change over two separate periods-aged 1018 years and $18-25$ years. ${ }^{4}$ Worryingly, while there was no accumulating impairment, lung function appeared to track parallel to controls-such that respiratory limitation seen in childhood remained in adult life, being more evident in those with BPD. Imaging has permitted a further insight into lung architecture with Wong et $a l^{5}$ demonstrating evidence of emphysematous change on CT scans in the majority of 19 young adults born with moderate to severe old BPD which was associated with worse spirometry and lower diffusion capacity. A subsequent study by the group demonstrated emphysematous change (or strictly, areas in which there are either or both of failure of lung development and lung destruction; an important lesson is not loosely to apply terms from adult medicine to new childhood problems, for fear of preventing clear thinking) in just under half the of 51 adult survivors of BPD. ${ }^{6}$

What becomes apparent is the small numbers in each of these studies, which is particularly striking in relation to the size of the at-risk population, given that nearly $8 \%$ of births in England and Wales are premature. ${ }^{7}$ Hence, extrapolation to the wider population at risk together with improved survival into adulthood poses the strongest warning signal of changing patterns in the adult respiratory clinic. How is it that we have failed to take this seriously? Currently, the reassurance that weaning off oxygen is a sign of improvement leads to most infants being discharged within the first 2 years of life unless there are coexistent neurodevelopmental issues. Even those under longer term paediatric care are lost as transitional care has not evolved. The lack of routine longer term follow-up has led to gaps in our knowledge. Those who do present later on with symptoms are likely to do so to non-specialists predominantly and hence may wrongly be diagnosed with asthma in childhood or COPD in later life. Of note, there is little evidence of ongoing airway inflammation in this group, ${ }^{8} 9$ meaning standard therapies for these diseases are likely to be useless; there may however be ongoing oxidative stress. ${ }^{10}$ Others may remain largely asymptomatic but have undiagnosed respiratory impairment, not least because those with comorbidities such as neurodevelopmental handicap and retinitis of prematurity may find difficulty in accessing care. The transition services for these adults are even more abysmal than in respiratory medicine. What a pity we have not followed the example of paediatric oncology and enrolled all these children in longitudinal, at least annual follow-up!

So, what are the implications of these manuscripts for adult respiratory physicians? Survivors from premature birth are increasing and they are at risk of respiratory morbidity. Walter et $a l^{11}$ demonstrated a greater risk of hospitalisation for respiratory illness in low birthweight adults. While, 
many may maintain sufficient respiratory reserve in health, the effect of further insults such as smoking is unknown-yet smoking among ex-preterm adults has been reported as greater than term controls. ${ }^{12}$ The pathophysiology of their premature lung disease is poorly characterised and is likely changing with successive cohorts-of especial note given current 20-25-year-olds represent the presurfactant era of neonatal care. Protocols for investigating these patients are lacking and thereby lies a huge potential for misdiagnosis and inappropriate medications. ${ }^{13}$ The longer term consequences are as yet unknown; for example, whether these survivors have a higher risk of lung cancer, something which will only be determined if we follow-up all these survivors.

There are also research implications which should be addressed. Received wisdom has been that there is no catch-up airway growth or alveolar regeneration. ${ }^{14}$ However, as Ira Gershwin might say, 'it ain't necessarily so!' A follow-up study of a cohort of preterm babies showed they had evidence of airflow obstruction aged 79 years, ${ }^{15}$ but normal spirometry aged 20 22 years. $^{12}$ Recent work in rhesus monkeys and humans suggests that alveolar growth actually continues until adult life. ${ }^{16-18}$ A group of admittedly not very preterm survivors aged 10-14 years studied with hyperpolarised helium had an alveolar size the same as in controls, implying alveolar catch-up growth. ${ }^{19}$ This chimes with the normal carbon monoxide transfer found even on extreme exercise in young adults, ${ }^{20}$ and the dramatic recovery that can be seen after apparently irretrievably destructive neonatal pneumonias. $^{21}$ These challenging studies suggest that there should not be too much clinical nihilism, but also that there are potential therapeutic targets which could lead to the exciting prospect of lung regrowth.

So what should our priorities be? They must include ongoing and renewed efforts to prevent premature births including reinforcing smoking legislation measures which when applied are associated with demonstrable improvements; ${ }^{22}$ we must follow-up all ex-preterm individuals, a difficult undertaking as services are hacked down (aka reorganised) in the name of 'efficiency'; and a multidisciplinary approach applied to the understanding of normal and abnormal lung growth is essential. Only thus can we develop novel treatment strategies. $^{23}$ Last, informing and education of healthcare professionals to take a more detailed history about neonatal and childhood factors, and understand and act on the answers, is paramount. ${ }^{1}$

Contributors $A B$ and $C E B$ both contributed to the drafting of this manuscript. Both $A B$ and $C E B$ have approved the final manuscript.

\section{Competing interests None.}

Provenance and peer review Commissioned; internally peer reviewed.

To cite Bolton CE, Bush A. Thorax 2013;68:707-708.

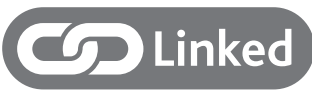

- http://dx.doi.org/10.1136/thoraxjnl-2012-203079

- http://dx.doi.org/10.1136/thoraxjnl-2012-202980

Thorax 2013;68:707-708

doi:10.1136/thoraxjnl-2013-203836

\section{REFERENCES}

1 Bolton CE, Bush A, Hurst JR, et al. Are early life factors considered when managing respiratory disease? A British Thoracic Society survey of current practice. Thorax 2012;67:1110.

2 Ben-Shlomo Y, Kuh D. A life course approach to chronic disease epidemiology: conceptual models, empirical challenges and interdisciplinary perspectives. Int J Epidemiol 2002;31:285-93.

3 Kotecha SJ, Edwards MO, Watkins WJ, et al. Effect of preterm birth on later FEV1: a systematic review and meta-analysis. Thorax 2013;68:760-6.

4 Vollsaeter M, Røksund OD, Eide GE, et al. Lung function after preterm birth: Development from mid-childhood to adulthood. Thorax 2013:68:767-76.

5 Wong PM, Lees AN, Louw J, et al. Emphysema in young adult survivors of moderate-to-severe bronchopulmonary dysplasia. Eur Respir J 2008;32:321-8.

6 Wong $\mathrm{P}$, Murray C, Louw J, et al. Adult bronchopulmonary dysplasia: computed tomography pulmonary findings. J Med Imaging Radiat Oncol 2011;55:373-8.

7 Office for National Statistics. Preterm births, preterm births data, press release based on 2005 data 2007 [cited 201325 May 2013]. http://www.ons.gov.uk/ ons/publications/re-reference-tables.html?edition=tcm $\% 3 A 77-50818$

8 Carraro S, Piacentini G, Lusiani M, et al. Exhaled air temperature in children with bronchopulmonary dysplasia. Pediatr Pulmonol 2010;45:1240-5.

9 Baraldi E, Bonetto G, Zacchello F, et al. Low exhaled nitric oxide in school-age children with bronchopulmonary dysplasia and airflow limitation. Am J Respir Crit Care Med 2005;171:68-72.

10 Filippone $M$, Bonetto G, Corradi M, et al. Evidence of unexpected oxidative stress in airways of adolescents born very pre-term. Eur Respir $J$ 2012;40:1253-9.

11 Walter EC, Ehlenbach WJ, Hotchkin DL, et al. Low birth weight and respiratory disease in adulthood: a population-based case-control study. Am J Respir Crit Care Med 2009;180:176-80.

12 Narang I, Rosenthal M, Cremonesini D, et al. Longitudinal evaluation of airway function 21 years after preterm birth. Am J Respir Crit Care Med 2008; 178:74-80.

13 Postma DS, Brusselle G, Bush A, et al. I have taken my umbrella, so of course it does not rain. Thorax 2012;67:88-9.

14 Narang I, Bush A. Early origins of chronic obstructive pulmonary disease. Semin Fetal Neonatal Med 2012;17:112-18.

15 Chan KN, Noble-Jamieson CM, Elliman A, et al. Lung function in children of low birth weight. Arch Dis Child 1989;64:1284-93.

16 Narayanan M, Owers-Bradley J, Beardsmore CS, et al. Alveolarization continues during childhood and adolescence: new evidence from helium-3 magnetic resonance. Am J Respir Crit Care Med 2012;185:186-91.

17 Hyde DM, Blozis SA, Avdalovic MV, et al. Alveoli increase in number but not size from birth to adulthood in rhesus monkeys. Am J Physiol Lung Cell Mol Physiol 2007;293:L570-9.

18 Butler JP, Loring SH, Patz S, et al. Evidence for adult lung growth in humans. $N$ Engl J Med 2012;367:244-7

19 Narayanan M, Beardsmore CS, Owers-Bradley J, et al. Catch-up Alveolarization in Ex-Preterm Children. Evidence from (3)He Magnetic Resonance. Am J Respir Crit Care Med 2013;187:1104-9.

20 Narang I, Bush A, Rosenthal M. Gas transfer and pulmonary blood flow at rest and during exercise in adults 21 years after preterm birth. Am J Respir Crit Care Med 2009:180:339-45.

21 Fang S, Bagtharia R, Kumar R, et al. Pneumatocoele and conjunctivitis in a newborn. Lancet 2004;364:1610.

22 Cox B, Martens E, Nemery $B$, et al. Impact of a stepwise introduction of smoke-free legislation on the rate of preterm births: analysis of routinely collected birth data. BMJ 2013;346:f441.

23 Krauss-Etschmann S, Bush A, Bellusci S, et al. Of flies, mice and men: a systematic approach to understanding the early life origins of chronic lung disease. Thorax 2013:68:380-4. 